\title{
Inhalt
}

\section{Heft 142}

\section{Fachwissenschaft}

Malte Steinbrink \& Philipp Aufenvenne (unter Mitarbeit von Jan-Berent Schmidt \& Max Pochadt) Integrative Geographiedidaktik? Versuch einer Positionsbestimmung der Fachdidaktik innerhalb der deutschsprachigen Geographie

\section{Fachdidaktik}

DAVID BURGER

Citizen Science, Partizipation und geographische Schulbildung

Nina Grünberg \& Christian Dorsch

Smarte Schüler/innen in der Smart City? Zur Bedeutung und Adaption eines Zukunftskonzepts in Schulbüchern

Helena Atteneder \& Ursula Maier-Rabler

Politische Bildung 2.0 - die "digitale Bildungsrevolution" am Beispiel "Polipedia.at"

AleXandra Budke, Miriam Kuckuck \& Maik Wienecke

Bedeutung der politischen Bildung im Geographieunterricht aus der Sicht von Geographielehrkräften

ITTA BAUER

"Vital Assemblages" als Konzept einer sozio-materiellen Geographiedidaktik und Unterrichtspraxis

Leif Mönter, Sabine Lippert \& Anna Gorges

Gemachte Armutsräume? Implikationen bei der Behandlung von Entwicklungsdisparitäten im geographischen Unterricht, vom Schulbuch zur Schülervorstellung

MARTIN DÜR

Lebensqualitätsvorstellungen als Basis einer Zusammenarbeit indischer und österreichischer Jugendlicher. Erste Ergebnisse eines Forschungsprojekts im Rahmen von Bildung für Nachhaltige Entwicklung

Gabriele Schrüfer, Gabriele Obermaier \& Sonja Schwarze

Raumwahrnehmung aus unterschiedlichen Perspektiven am Beispiel Tansania - Empirische Untersuchungen und Konsequenzen für den Geographieunterricht

\section{Unterrichtspraxis}

Katja Marso, Zoran Jovic, Christoph Milchrahm, Josef Rathmair \& Stefan Padberg Interesse Macht Raum - auch im Krieg nebenan. Und wie siehst du das?

\section{Heft 143}

\section{Fachdidaktik}

SteFAn HINSCH

Gegen die Sinuskurve - Zur Theorie des Konjunkturzyklus und seiner Darstellung in österreichischen Schulbüchern 
Geographiedidaktische Überlegungen zu Basiskonzepten des Unterrichtsfachs GWK unter kritischem Bezug auf sozial-konstruktivistische Raumkonzepte und digitale Geomedien - ein Diskussionsbeitrag

Anne-Kathrin Lindau, Daniela Hottenroth \& Martin Lindner Wildnisbildung als Möglichkeit zur Professionalisierung zukünftiger Geographielehrer/innen - ein neuer Ansatz zur Bildung für nachhaltige Entwicklung?

Steffen Höhnle \& Jan Christoph Schubert

Hindernisse für den Einsatz naturwissenschaftlicher Arbeitsweisen im Geographieunterricht aus Studierendenperspektive - Ausgewählte Ergebnisse einer empirischen Studie mit Lehramtsstudierenden

Raimund DitTer \& AleXANder Siegmund

Lernen mit digitalen Satellitenbildern - eine genderspezifische Analyse

\section{Unterrichtspraxis}

André Hermes \& Miriam Kuckuck

Digitale Lehrpfade selbstständig entwickeln - Die App Actionbound als Medium für den Geographieunterricht zur Erkundung außerschulische Lernorte

Hans-Peter Steyrer \& Sylke Hilberg

Sand und Wasser: Analogieexperimente zur Geologie im Schulunterricht

\section{Kontrapunkt / Service}

Tobias Hinterseer, Florian Preisig \& Michaela Schmidt

Eckpunkte für die ökonomische Bildung im schulischen Kontext - Gemeinsamkeiten zwischen schulischer und außerschulischer Bildungsarbeit im Bereich der ökonomischen Bildung

Stefan Padberg, Herbert Pichler, Christiane Hintermann \& Stefan Baumann

Flucht und Migration bewegt Schüler/innen, Studierende und Lehrpersonen! Geographiedidaktik und Geographieunterricht für Menschenrechte und gegen Rassismus 